\title{
Lorentz Force in Special Relativity theory
}

\author{
Sangwha-Yi* \\ Department of Math, Taejon University 300-716, South Korea
}

*Corresponding Author: Sangwha-Yi, Department of Math, Taejon University 300-716, South Korea

Abstract: In the special relativity theory, we know how Lorentz, 4-force is invariant in special relativity theory.

Keywords: Special relativity theory, Lorentz force, Electro-magnetic field transformation

PACS Number: 03.30, 41.20

\section{INTRODUCTION}

Our article's aim is that we tell how Lorentz 4-force is invariant by electro-magnetic field transformations in special relativity theory.

At first, the coordinate transformation is in Special relativity theory,

$c t=\gamma\left(c t^{\prime}+\frac{v}{c} x^{\prime}\right), x=\gamma\left(x^{\prime}+\frac{v}{c} c t^{\prime}\right), y=y^{\prime}, z=z^{\prime}$

Therefore, Minkowski 4-force is in Special Relativity theory[5]

$$
\begin{aligned}
& f^{0}=m_{0} c \frac{d^{2} t}{d \tau^{2}}=m_{0} \gamma\left(c \frac{d^{2} t^{\prime}}{d \tau^{2}}+\frac{v}{c} \frac{d^{2} x^{\prime}}{d \tau^{2}}\right)=\gamma\left(f^{10}+\frac{v}{c} f^{\prime \prime}\right), \\
& f^{1}=m_{0} \frac{d^{2} x}{d \tau^{2}}=m_{0} \gamma\left(\frac{d^{2} x^{\prime}}{d \tau^{2}}+\frac{v}{c} \frac{c d^{2} t^{\prime}}{d \tau^{2}}\right)=\gamma\left(f^{\prime \prime}+\frac{v}{c} f^{0}\right) \\
& f^{10}=m_{0} c \frac{d^{2} t^{\prime}}{d \tau^{2}}, f^{\prime \prime}=m \frac{d^{2} x^{\prime}}{d \tau^{2}} \\
& f^{2}=m_{0} \frac{d^{2} y}{d \tau^{2}}=m_{0} \frac{d^{2} y^{\prime}}{d \tau^{2}}=f^{2}, f^{3}=m_{0} \frac{d^{2} z}{d \tau^{2}}=m_{0} \frac{d^{2} z^{\prime}}{d \tau^{2}}=f^{B}
\end{aligned}
$$

Hence, in inertial frame, Lorentz 4-force is

$$
\begin{aligned}
& F^{0}=m_{0} \frac{d}{d t}\left(\frac{c d t}{d \tau}\right)=q \frac{\vec{u}}{c} \cdot \vec{E} \\
& \vec{F}=m_{\circ} \frac{d}{d t}\left(\frac{d \vec{x}}{d \tau}\right)=q\left[\vec{E}+\frac{\vec{u}}{c} \times \vec{B}\right], \quad \vec{u}=\frac{d \vec{x}}{d t} \\
& F^{10}=m_{0} \frac{d}{d t^{\prime}}\left(\frac{c d t^{\prime}}{d \tau}\right)=q \frac{\vec{u}^{\prime}}{c} \cdot \vec{E}^{\prime} \\
& \vec{F}^{\prime}=m_{0} \frac{d}{d t^{\prime}}\left(\frac{d \vec{x}^{\prime}}{d \tau}\right)=q\left[\vec{E}^{\prime}+\frac{\vec{u}^{\prime}}{c} \times \vec{B}^{\prime}\right], \quad \vec{u}^{\prime}=\frac{d \vec{x}^{\prime}}{d t^{\prime}}
\end{aligned}
$$

\section{INVARIANT LORENTZ 4-FORCE IN INERTIAL FRAME}

In this time, Minkowski 4-force is in inertial frame. 


$$
\begin{aligned}
& f^{0}=m_{0} c \frac{d^{2} t}{d \tau^{2}}=q \frac{\vec{u}}{c} \cdot \vec{E} \frac{d t}{d \tau} \quad, \vec{u}=\frac{d \vec{x}}{d t}, \vec{u}^{\prime}=\frac{d \vec{x}^{\prime}}{d t^{\prime}} \\
& =m_{0} \gamma\left(c \frac{d^{2} t^{\prime}}{d \tau^{2}}+\frac{v}{c} \frac{d^{2} x^{\prime}}{d \tau^{2}}\right)=\gamma\left(f^{0}+\frac{v}{c} f^{\prime \prime}\right) \\
& =\gamma q \frac{\vec{u}^{\prime}}{c} \cdot \vec{E}^{\prime} \frac{d t^{\prime}}{d \tau}+\gamma \frac{v}{c}\left[q E_{x}{ }^{\prime}+q \frac{1}{c}\left(u_{y}{ }^{\prime} B_{z}{ }^{\prime}-u_{z}^{\prime} B_{y}^{\prime}\right)\right] \frac{d t^{\prime}}{d \tau} \\
& f^{1}=m_{0} \frac{d^{2} x}{d \tau^{2}}=m_{0} \gamma\left(\frac{d^{2} x^{\prime}}{d \tau^{2}}+\frac{v}{c} \frac{c d^{2} t^{\prime}}{d \tau^{2}}\right)=\gamma\left(f^{\prime \prime}+\frac{v}{c} f^{0}\right) \\
& =\gamma\left[q E_{x}{ }^{\prime}+q \frac{1}{c}\left(u_{y}{ }^{\prime} B_{z}{ }^{\prime}-u_{z}{ }^{\prime} B_{y}{ }^{\prime}\right)\right] \frac{d t^{\prime}}{d \tau}+\gamma \frac{v}{c}\left(q \frac{\vec{u}^{\prime}}{c} \cdot \vec{E}^{\prime}\right) \frac{d t^{\prime}}{d \tau} \\
& f^{\prime 0}=m_{0} c \frac{d^{2} t^{\prime}}{d \tau^{2}}=q \frac{\vec{u}^{\prime}}{c} \cdot \vec{E}^{\prime} \frac{d t^{\prime}}{d \tau}, f^{\prime \prime}=m_{0} \frac{d^{2} x^{\prime}}{d \tau^{2}}=q\left[E_{x}{ }^{\prime}+\frac{1}{c}\left(u_{y}^{\prime} B_{z}^{\prime}-u_{z}^{\prime} B_{y}^{\prime}\right)\right] \frac{d t^{\prime}}{d \tau}
\end{aligned}
$$

In This Time, The Transformation Of Electromagnetic Field Is In Special Relativity Theory.

$$
\begin{aligned}
& E_{x}=E_{x}{ }_{x}, E_{y}=\gamma E^{\prime}{ }_{y}+\gamma \frac{V}{c} B^{\prime}{ }_{z}, E_{z}=\gamma E_{z}{ }_{z}-\gamma \frac{v}{c} B^{\prime}{ }_{y} \\
& B_{x}=B_{x}{ }_{x}, B_{y}=\gamma B^{\prime}{ }_{y}-\gamma \frac{V}{c} E_{z}^{\prime}, B_{z}=\gamma B_{z}{ }_{z}+\gamma \frac{V}{c} E^{\prime}{ }_{y}
\end{aligned}
$$

Hence, If We Calculate For Proving Invariant Of Lorentz 4-Force By Eq(10),Eq(11),Eq(12),Eq(13),

T-Component Is

$$
\begin{aligned}
& f^{0}=m_{0} c \frac{d^{2} t}{d \tau^{2}}=q \frac{\vec{u}}{c} \cdot \vec{E} \frac{d t}{d \tau} \\
& =q \frac{1}{c}\left(u_{x} E_{x}+u_{y} E_{y}+u_{z} E_{z}\right) \frac{d t}{d \tau} \\
& =q \frac{1}{c}\left[\left(\frac{u_{x}{ }^{\prime}+v}{1+\frac{u_{x}{ }^{\prime} v}{c^{2}}}\right) E^{\prime}{ }_{x}+\frac{u_{y}{ }^{\prime}}{\gamma\left(1+\frac{u_{x}{ }^{\prime} v}{c^{2}}\right)} \gamma\left(E_{y}^{\prime}+\frac{v}{c} B_{z}^{\prime}\right)+\frac{u_{z}^{\prime}}{\gamma\left(1+\frac{u_{x}{ }^{\prime} v}{c^{2}}\right)} \gamma^{\prime}\left(E_{z}^{\prime}-\frac{v}{c} B_{y}^{\prime}\right)\right] \\
& \times \gamma \frac{d t^{\prime}}{d \tau}\left(1+\frac{u_{x}{ }^{\prime} v}{c^{2}}\right) \\
& =\gamma q \frac{1}{c}\left(u_{x} E_{x}{ }^{\prime}+u_{y}{ }^{\prime} E_{y}{ }^{\prime}+u_{z}{ }^{\prime} E_{z}{ }^{\prime}\right) \frac{d t^{\prime}}{d \tau}+\gamma \frac{v}{c}\left[q E_{x}{ }^{\prime}+q \frac{1}{c}\left(u_{y}{ }^{\prime} B_{z}{ }^{\prime}-u_{z}{ }^{\prime} B_{y}{ }^{\prime}\right)\right] \frac{d t^{\prime}}{d \tau} \\
& =\gamma\left(f^{\prime 0}+\frac{v}{c} f^{\prime \prime}\right)
\end{aligned}
$$

$\mathrm{X}$-component is

$$
\begin{aligned}
& f^{1}=m_{0} \frac{d^{2} x}{d \tau^{2}}=\left[q E_{x}+q \frac{1}{c}\left(u_{y} B_{z}-u_{z} B_{y}\right)\right] \frac{d t}{d \tau} \\
& =\left[q E_{x}{ }_{x}+q\left(\frac{1}{c}\left(\frac{u_{y}{ }^{\prime}}{\gamma\left(1+\frac{u_{x}{ }^{\prime} v}{c^{2}}\right)}\right) \gamma\left(B^{\prime}{ }_{z}+\frac{v}{c} E_{y}^{\prime}\right)-\frac{u_{z}{ }^{\prime}}{\gamma\left(1+\frac{u_{x}{ }^{\prime} v}{c^{2}}\right)} \gamma\left(B^{\prime}{ }_{y}-\frac{v}{c} E_{z}^{\prime}\right)\right)\right] \\
& \times \gamma \frac{d t^{\prime}}{d \tau}\left(1+\frac{u_{x}{ }^{\prime} v}{c^{2}}\right)
\end{aligned}
$$




$$
\begin{aligned}
& =\gamma\left[q E_{x}{ }^{\prime}+q \frac{1}{c}\left(u_{y}{ }^{\prime} B_{z}{ }^{\prime}-u_{z}{ }^{\prime} B_{y}{ }^{\prime}\right)\right] \frac{d t^{\prime}}{d \tau}+\gamma \frac{v}{c}\left[q \frac{1}{c}\left(u_{x}{ }^{\prime} E_{x}{ }^{\prime}+u_{y}{ }^{\prime} E_{y}{ }^{\prime}+u_{z}{ }^{\prime} E_{z}{ }^{\prime}\right)\right] \frac{d t^{\prime}}{d \tau} \\
& =\gamma\left(f^{\prime \prime}+\frac{v}{c} f^{\prime 0}\right)
\end{aligned}
$$

Y-component is

$$
\begin{aligned}
& f^{2}=m_{0} \frac{d^{2} y}{d \tau^{2}}=\left[q E_{y}+q \frac{1}{c}\left(u_{z} B_{x}-u_{x} B_{z}\right)\right] \frac{d t}{d \tau} \\
& =\left[q \gamma\left(E_{y}^{\prime}+\frac{v}{c} B_{z}^{\prime}\right)+q\left(\frac{1}{c}\left(\frac{u_{z}{ }^{\prime}}{\gamma\left(1+\frac{u_{x}{ }^{\prime} v}{c^{2}}\right)}\right) B_{x}^{\prime}-\frac{u_{x}{ }^{\prime}+v}{1+\frac{u_{x}{ }^{\prime} v}{c^{2}}} \gamma\left(B^{\prime}{ }_{z}+\frac{v}{c} E_{y}^{\prime}\right)\right)\right] \\
& \times \gamma \frac{d t^{\prime}}{d \tau}\left(1+\frac{u_{x}{ }^{\prime} v}{c^{2}}\right) \\
& =\left[q E_{y}{ }^{\prime}+q \frac{1}{c}\left(u_{z}{ }^{\prime} B_{x}{ }^{\prime}-u_{x}{ }^{\prime} B_{z}{ }^{\prime}\right)\right] \frac{d t^{\prime}}{d \tau}=f^{R}
\end{aligned}
$$

Z-component is

$$
\begin{aligned}
& f^{3}=m_{0} \frac{d^{2} z}{d \tau^{2}}=\left[q E_{z}+q \frac{1}{c}\left(u_{x} B_{y}-u_{y} B_{x}\right)\right] \frac{d t}{d \tau} \\
& =\left[q \gamma\left(E_{z}^{\prime}-\frac{v}{c} B_{y}^{\prime}\right)+q\left(\frac{1}{c}\left(\frac{u_{x}{ }^{\prime}+v}{1+\frac{u_{x}{ }^{\prime} v}{c^{2}}}\right) \gamma\left(B^{\prime}{ }_{y}-\frac{v}{c} E_{z}^{\prime}\right)-\frac{u_{y}^{\prime}}{\gamma\left(1+\frac{u_{x}{ }^{\prime} v}{c^{2}}\right)} B_{x}^{\prime}\right)\right] \\
& \times \gamma \frac{d t^{\prime}}{d \tau}\left(1+\frac{u_{x}{ }^{\prime} v}{c^{2}}\right) \\
& =\left[q E_{z}{ }^{\prime}+q \frac{1}{c}\left(u_{x}{ }^{\prime} B_{y}{ }^{\prime}-u_{y}{ }^{\prime} B_{x}{ }^{\prime}\right)\right] \frac{d t^{\prime}}{d \tau}=f^{B}
\end{aligned}
$$

\section{CONCLUSION}

We know Lorentz 4-force is invariant by the Lorentz transformation in special relativity theory .

\section{REFERENCES}

[1] W.Rindler, Am.J.Phys.34.1174(1966)

[2 ]A.Miller, Albert Einstein's Special Theory of Relativity(Addison-Wesley Publishing Co., Inc., 1981)

[3] W.Rindler, Special Relativity(2nd ed., Oliver and Boyd, Edinburg,1966)

[4] A. Einstein, “ Zur Elektrodynamik bewegter K"orper”, Annalen der Physik. 17:891(1905)

[5] D.J. Griffith,” Introduction To Electrodynamics”,(2nd ed.,Prentice Hall,Inc.1981)

Citation: Sangwha-Yi, (2019). Lorentz Force in Special Relativity theory. International Journal of Advanced Research in Physical Science (IJARPS) 6(12), pp.8-10, 2019.

Copyright: () 2019 Authors, This is an open-access article distributed under the terms of the Creative Commons Attribution License, which permits unrestricted use, distribution, and reproduction in any medium, provided the original author and source are credited. 